Trascender, Contabilidad y Gestión. Vol. 6, Núm. 17 (mayo - agosto del 2021).

Universidad de Sonora. Departamento de Contabilidad. México.

ISSN: 2448-6388. Reserva de Derechos 04-2015-04172070800-203.

\title{
Evaluación del programa emprendedor de Ingeniería en Gestión Empresarial en Tecnológico Superior de El Mante
}

\author{
Evaluation of Business Management Engineering entrepreneurship program at Tecnológico \\ Superior de El Mante \\ Daniel Eduardo Paz Pérez ${ }^{1}$
}

Recibido: 28 de enero de 2021.

Aceptado: 10 de marzo de 2021.

DOI: https://doi.org/10.36791/tcg.v0i17.100

JEL: A22. Enseñanza universitaria. L26. Emprendedores. M13. Creación de empresas.

\section{Resumen}

En un trabajo exploratorio y descriptivo del tipo transversal se buscó reconocer las actividades que promueven el emprendimiento en los estudiantes de la carrera de Ingeniería en Gestión Empresarial ofrecida desde el año 2009 por un instituto regional dependiente del Tecnológico Nacional de México; a través de un análisis bibliométrico utilizando diferentes bases de datos científicas se encontraron estudios recientes referidos en forma exclusiva a los siguientes temas: "actividades de emprendimiento", "metodologías de emprendimiento” y "programas de emprendimiento"; el fenómeno se analizó desde la Teoría General de Sistemas utilizando el método documental sistémico-estructural-funcional y técnicas cualitativas de análisis como grupo nominal para identificar las competencias emprendedoras del perfil de egreso y las actividades que promueven el emprendimiento en los estudiantes; el resultado permite identificar dos competencias emprendedoras y las actividades que la academia realiza para su

${ }^{1}$ Daniel Eduardo Paz Pérez. Contador Público. Doctor en Materia Fiscal. Docente Investigador en Línea de Investigación Educativa. Academia de Gestión Empresarial, Tecnológico Nacional de México / ITS de El Mante. Ciudad Mante, Tamaulipas, México. Correo: depaz@itsmante.edu.mx. ORCID: https://orcid.org/0000-0003-0511-2776

Vol. 6, núm. 17 / mayo - agosto del 2021 
promoción. Se obtienen conclusiones sobre la insuficiencia de las actividades incluidas en el programa emprendedor del ITSMante para la formación del perfil que se busca en los estudiantes de la carrera y se realizan propuestas de incorporación a dicho programa.

Palabras clave: docencia, emprendimiento, metodologías, programas, gestión.

\section{Abstract}

In an exploratory and descriptive work of transversal type, it was sought to recognize activities that promote entrepreneurship in Business Management Engineering' students, offered since 2009 by a regional institute at Tecnológico Nacional de México; via a bibliometric analysis using different scientific databases, recent studies were found exclusively referring to the following topics: "entrepreneurship activities", "entrepreneurship methodologies" and "entrepreneurship programs"; the phenomenon was analyzed from the General Theory of Systems using the systemic-structural-functional documentary method and qualitative analysis techniques as nominal group to identify the graduate profile's entrepreneurial competences, as well as activities that promote entrepreneurship in students; the result allows to identify two entrepreneurial competencies and the activities that promote it. It is concluded about insufficiency of the activities included in ITSMante entrepreneurial program to build such profile sought in BME students, as well as proposals to improve the program.

Keywords: Teaching. Entrepreneurship. Methods. Programs. Management.

\section{Introducción}

El estudio del emprendimiento es una actividad importante para entender a la sociedad moderna, cada vez más preocupada por los problemas económicos y de desempleo mundial, pero inicialmente no existe en la literatura actual una definición consensuada del término emprendedor (Fuentes García y Sánchez Cañizares, 2010; Herrera Guerra y Montoya Restrepo, 2013; Marulanda Valencia, Montoya Restrepo y Vélez Restrepo, 2014; Moriano León, Palací Descals y Morales Domínguez, 2006); de hecho, es un fenómeno en constante investigación, del cual dan cuenta numerosos trabajos que versan sobre su concepción y evolución histórica, las múltiples teorías de la intención emprendedora y los principales atributos del emprendedor, a partir de numerosos estudios analizados (Il Sung Park y Duarte Masi, 2015; Valencia Arias, Montoya Restrepo y Montoya Restrepo, 2015).

Algunas investigaciones reconocen al emprendimiento como una sub disciplina de la administración estratégica, razón por la que suele ser incluido en las actividades de enseñanza de nivel superior y particularmente en las escuelas de administración, en donde se fomentan las habilidades emprendedoras en los jóvenes universitarios mediante diversas actividades (Bedoya Villa y Arango Alzate, 2017; Herrera Guerra y Montoya Restrepo, 2013; Vargas Hernández y Campos Álvarez, 2011). Es por ello que las universidades juegan un papel importante en la formación del talento emprendedor y de la generación de nuevas empresas a partir de la implementación de programas que promueven la aproximación al mundo empresarial, la vinculación con la industria y la colaboración con el sector social. 
En la actualidad, la mayoría de las universidades incluyen el factor del emprendimiento en la formación estudiantil con la finalidad de que sus egresados puedan crear su propio negocio y auto emplearse al finalizar su formación, ya que está visto que el emprendimiento es una herramienta para reducir la pobreza, además de promover el desarrollo sustentable al tiempo que revitaliza el entorno social (Garzozi, Messina, Moncada, Ochoa, Ilabel y Zambrano, 2014; Leite, Correia, Sánchez-Fernández y Leite, 2015), pero no todas las actividades que se proponen con tal fin logran despertar el espíritu emprendedor de los estudiantes. La integración de un portafolio de actividades emprendedoras es deseable para las instituciones educativas, ya que permite reforzar las competencias con las que los egresados incursionarán en la industria, desempeñando mejor las funciones y actividades que son acordes a su perfil (Almanza Jiménez y VargasHernández, 2015); de hecho, un problema recurrente en los sistema educativos latinoamericanos es la baja intención emprendedora de los estudiantes (Moriano León et al., 2006), de ahí la importancia de este trabajo. Las preguntas que detonan esta investigación son: ¿cuáles actividades se promueven en las universidades para desarrollar el talento emprendedor? y ¿qué actividades se consideran más efectivas para lograrlo?

El presente estudio se llevó a cabo en el Instituto Tecnológico Superior de El Mante (ITSMante), perteneciente al Tecnológico Nacional de México que agrupa 260 institutos tecnológicos federales y descentralizados; en esta institución se ofrece la carrera de Ingeniería en Gestión Empresarial desde el año 2010, con una matrícula local de 240 alumnos en el año 2020 cursando estudios en los diferentes niveles o ciclos; los docentes de la Academia de esta carrera participaron en la evaluación del programa emprendedor a invitación del docente investigador.

La primera sección de este trabajo se dedica al estudio del fenómeno del emprendimiento a partir de la literatura reciente y en particular el de tipo universitario; en la segunda sección se profundiza en el perfil y las características del emprendedor; en la tercera sección se revisan los programas de fomento emprendedor en las universidades; a continuación se describe el enfoque metodológico utilizado, el cual se basó en técnicas cualitativas, para mostrar finalmente los resultados encontrados y las conclusiones del autor; en el último apartado se realiza la discusión de las actividades que fomentan el emprendimiento en las instituciones educativas superiores.

\section{El emprendimiento}

El estudio de los nuevos negocios es una actividad bajo la lupa, ha sido tema de constante investigación y a partir de él se han generado teorías y postulados; al respecto, Leite et al. (2015) nos dicen que la generación de riqueza ya no proviene del uso del trabajo y del capital sino del conocimiento, el cual posibilita la agregación de valor en la industria moderna y contribuye a la producción de riqueza en el mundo, por lo cual ahora se concibe al conocimiento como el nuevo sustrato que nutre el emprendimiento de los negocios en el siglo XXI; este fenómeno también es visto con detenimiento por la academia, en razón de que su estudio a partir del comportamiento de los jóvenes universitarios es altamente sugerido por la similitud de la intención emprendedora que tienen éstos con los jóvenes no universitarios y que permite extrapolar sus resultados a otros ámbitos, de allí la prevalencia de su investigación (Valencia Arias et al., 2015). 
El emprendimiento también es visto como un ecosistema que puede ser estimulado en una combinación de personas, recursos disponibles a nivel global o regional y otros sectores mostrados en la figura 1 que enmarcan y condicionan su desempeño (Ács, Szerb, Lafuente y Márkus, 2020). En dicha estructura, las capacidades y aspiraciones empresariales de cada jóven son estimulados por la infraestructura, el mercado, el sistema educativo y demás sectores, hasta que logran ser motivados a convertirse en nuevos emprendedores por las actitudes que prevalecen en el grupo poblacional, de ahí la importancia de todo el ecosistema en su conjunto.

\section{Figura 1}

\section{Estructura del ecosistema emprendedor}

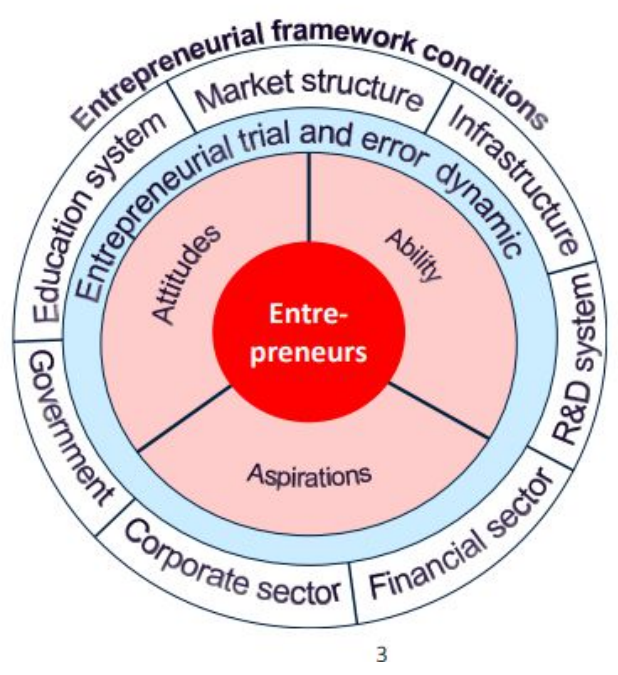

Fuente: Ács, Szerb, Lafuente y Márkus (2020).

De acuerdo con Garzozi et al. (2014), existen diferentes tipos de emprendimientos, los cuales se agrupan en las siguientes tres categorías: en función a la actividad, pueden ser emprendimientos de base abierta, de base cultural, de base tecnológica (EBT), dinámicos o startups; en función al objetivo, pueden ser de base social o inclusivos; y en función a su origen, pueden ser spin-off, que son empresas creadas para explotar comercialmente los resultados a partir del trabajo e investigaciones que surgen en las universidades. Esta actividad emprendedora es realizada con frecuencia por jóvenes universitarios que llevan a la praxis económica los conocimientos empresariales que les han sido impartidos, impulsados por el espíritu emprendedor que despierta en ellos, bien porque éste fue promovido por actividades realizadas $e x$ profeso por la universidad o porque se encuentran en una etapa de su vida en la que sus aspiraciones y motivaciones refuerzan dicha cualidad; podemos entender al espíritu emprendedor como aquellos rasgos de la personalidad que se manifiestan en la forma de pensar y actuar frente a situaciones de oportunidad (Carlos Ornelas, Contreras González, Silva Olvera y 
Liquidano Rodríguez, 2015; Sánchez Rodríguez, Hernández Herrera y Jiménez García, 2016; Valencia Arias et al., 2015).

El perfil emprendedor es una característica innata de algunas personas, pero también puede ser formado o fomentado, apuntalando desde la educación inicial hasta la que ofrecen las instituciones de nivel superior aquellos factores que lo desarrollan; durante su estadía en éstas últimas, los estudiantes son involucrados en actividades diseñadas para conocer situaciones concretas que los aproximen al mundo real donde ofrecerán sus servicios, lo cual permite una mejor trayectoria profesional, para ello es tarea de la academia de cada carrera diseñar e implementar las actividades más acordes con el perfil que se pretende formar en los estudiantes; de igual forma, se considera que las universidades también son emprendedoras cuando realizan actividades de triple hélice que vinculan la educación y la investigación con otras asociaciones, redes y organizaciones públicas y privadas que promueven la innovación, y que aquellas trasladan al estudiante en su formación emprendedora (Guerrero y Urbano, 2012; Paños Castro, 2017), pero también se conoce que el déficit emprendedor de estas instituciones es asimismo la primer causa de inhibición en la generación de spin-offs universitarias (Zachman, López y Redchuk, 2015). En particular, los programas educativos de las carreras administrativas son los que contemplan más materias relacionadas con el emprendimiento (38\% en las universidades mexicanas) así también como programas emprendedores (35\%), por encima de las demás carreras (Moreno Zacarías, Espíritu Olmos y Priego Huertas, 2013).

\section{Perfil y características del emprendedor}

En la literatura podemos encontrar muchas definiciones de lo que es un emprendedor y la mayoría de los autores consideran al término francés entreprendre (que "significa hacerse cargo") como la fuente original del término entrepreneur más utilizado en las publicaciones científicas; Fuentes García y Sánchez Cañizares (2010) realizaron un análisis bibliométrico sobre el tema y no encuentran una definición única de dicho término, y notan que se le caracteriza como el empresario o quien comienza una empresa pero también el que la dirige, incluso aquel que lleva adelante un proyecto sin ánimo de lucro; formulando una propia definición, Herrera Guerra y Montoya Restrepo acotan que emprendedor es "la persona que crea empresas asumiendo riesgos” (2013, p. 21). De igual forma, Marulanda Valencia et al., (2014) señalan la falta de consenso para establecer un perfil único del emprendedor, pero recopilan de algunos autores los elementos detonadores del emprendimiento, encontrando que uno de los más cruciales son las aspiraciones o motivaciones del emprendedor, así como sus competencias y recursos; en un estudio más amplio del tema, el reporte anual creado por el Instituto del Emprendimiento Global y Desarrollo (Ács et al., 2020), propone 14 pilares del perfil del emprendedor emergente, agrupados en las 3 categorías siguientes:

1. Los 5 pilares de la actitud emprendedora: 1) la oportunidad empresarial, que es la percepción del individuo al identificar buenas oportunidades de crear un negocio en función de la regulación del gobierno y el mercado; 2) las habilidades, que suelen ser reforzadas con la educación

Vol. 6, núm. 17 / mayo - agosto del 2021 
universitaria, y que serán mejor desarrolladas que los conocimientos empíricos en los que se basan muchos jóvenes para emprender; 3) la aceptación o aversión al riesgo; 4) networking, la capacidad de relación con otros para compartir su conocimiento empresarial; 5) el soporte cultural, llamando así a la forma en la que la sociedad en su conjunto aprecia y promueve el emprendimiento, o lo rechaza y empuja al jóven a buscar alternativas tradicionales como la educación formal;

2. Los 4 pilares de la habilidad emprendedora: 6) el emprendimiento oportuno, la facilidad que pueda encontrarse en la burocracia para iniciar un nuevo negocio; 7) el nivel de absorción de tecnología que prevalece en el entorno; 8) el capital humano, la experiencia, innovación y salud de la fuerza de trabajo; 9) la competencia, vista desde el mercado y las restricciones legales;

3. Los 5 pilares de la aspiración emprendedora: 10) la innovación del producto que ofrecerá el nuevo empresario; 11) la innovación del proceso, aplicando tecnología de reciente creación; 12) llamado alto crecimiento, identifica los negocios que planean tener más de 10 empleados y y cuya intención es crecer al 50\% en los próximos 5 años, apoyados en un plan estratégico; 13) la internacionalización, la identificación de la intención exportadora del emprendedor; 14) el capital de riesgo, preferentemente emprender con capital propio y no con deudas.

Al emprendedor se le considera también una persona creativa, persistente, innovadora, generadora de empleo y transformadora de recursos; algunos atributos determinantes de la actividad emprendedora son los factores sociodemográficos como el género, la edad, la familia, el nivel educativo, la experiencia laboral, así como otros factores personales tales como la disciplina, la necesidad de logro, el optimismo, la responsabilidad hacia el trabajo o el temor (Fuentes García y Sánchez Cañizares, 2010; Moriano et al., 2006).

\section{Programas de fomento emprendedor en las universidades}

Como parte de sus actividades regulares, las instituciones académicas de nivel superior fomentan el emprendimiento entre los estudiantes, pero tales actividades deben ser diseñadas e impartidas en forma paralela a los programas oficiales que contemplan actividades orientadas solamente al conocimiento científico, a fin de lograr una mayor eficacia en los resultados (Leite et al., 2015; Marulanda Valencia et al., 2014); también se ha comprobado que si se promueven adecuadamente los programas y se hacen evidentes las ventajas que representa el emprendimiento de nuevos negocios se podrá influir positivamente en la actitud de los jóvenes universitarios a la hora de planear sus propios emprendimientos (Rueda Sampedro, FernándezLaviada y Herrero Crespo, 2013). Esta educación emprendedora moderna tiene tres enfoques, según Sánchez García, Ward, Hernández y Florez (2017): educación sobre el emprendimiento, para conocer las teorías conceptuales; educación para emprender, con una perspectiva de capacitación teórico-práctica; y educación a través de emprendimiento, en el que se experimenta al tiempo que se aprende; mientras la primera variante de la educación emprendedora es muy utilizada en los centros educativos para trasladar al educando el conocimiento inicial, las 2 últimas son las más reconocidas cuando los estudiantes evalúan su formación universitaria.

Como se ha señalado antes, las instituciones de educación superior integran en sus actividades 
educativas diferentes programas de fomento emprendedor bajo la premisa de que tales actividades son críticas para desarrollar en el educando las competencias necesarias para la creación de empresas, luego entonces tales instituciones funcionan como incubadoras de proyectos de negocios, aunque a la hora de medir los resultados algunos programas presenten mayor efectividad que otros (Guerrero y Urbano, 2012; Sánchez-García y Hernández-Sánchez, 2016); en otro trabajo, Vargas Hernández y Campos Álvarez (2011) nos ayudan a reconocer los objetivos que se plantean algunas de las instituciones más reconocidas del país al implementar dichos programas, encontrando similitud entre algunos como el Programa del Centro UNAM, el Programa EmprendeTEC del ITSM, el Programa CIEBT del IPN y el Programa Asociación de empresas del ITAM, en los cuales el propósito ulterior es el desarrollo del alumno en la cultura emprendedora para la creación de empresas.

También Moreno Zacarías et al. (2013) en su investigación sobre universidades mexicanas encuentran que los apoyos a la formación del estudiante son principalmente en la forma de las incubadoras de negocios (46\%), centros de formación de negocios (13,5\%) y programas de emprendedores (37,8\%); respecto al funcionamiento de estos últimos, Vargas Hernández y Campos Álvarez (2011) no encuentran indicadores recientes de la eficiencia de dichos programas y proponen estos tres indicadores para su evaluación: el grado de desarrollo de las habilidades emprendedoras de los alumnos, la cantidad de empresas creadas por alumnos o egresados de las universidades y el número de egresados (intrapreneurs) trabajando en niveles directivos en organizaciones existentes; de igual forma, De la Torre Cruz, Luis Rico, Escolar Llamazares,
Palmero Cámara y Jiménez Eguizábal (2016) afirman que la función primordial de los programas de apoyo al talento emprendedor es la conformación de redes de emprendimiento, en las cuales las actividades se centran en la creación de alianzas y comunidades virtuales, así como en la organización de foros, tertulias, premios, jornadas, prácticas y galas para el intercambio de experiencias que posibiliten la solución de problemas.

En ese mismo contexto, Guerrero y Urbano (2012) encuentran que tales programas y prácticas emprendedoras universitarias ofrecen resultados que contribuyen al desarrollo regional, y que pueden relacionarse con el número de empresas de nueva creación, nuevas patentes, incremento del empleo, la riqueza y el prestigio de la universidad; sin embargo, aunque en la literatura se cita con frecuencia la educación emprendedora, no se suele detallar qué metodologías utilizar para llevarla a cabo, por lo que la elección de las herramientas efectivas para el logro de tales competencias es una labor difícil para el profesorado (Paños Castro, 2017).

\section{Materiales y métodos}

El presente es un estudio descriptivo de tipo exploratorio y transversal, dado que la carrera de Ingeniería en Gestión Empresarial (IGE) que ofrece el Tecnológico Nacional de México es de reciente creación en 2009; con un enfoque cualitativo se buscó identificar las actividades organizadas por la Academia de dicha carrera que fomentan el emprendimiento de los estudiantes de la carrera, en el Instituto Tecnológico Superior de El Mante; para su ejecución fue necesario un trabajo bibliométrico consistente en la revisión de diversas bases de datos científicas con el fin de identificar los estudios recientes referidos al tema, a fin 
de construir un marco de referencia exclusivo sobre los siguientes tópicos: "actividades de emprendimiento", "metodologías de emprendimiento" y "programas de emprendimiento”; para analizar el fenómeno desde la perspectiva de las instituciones educativas se abordó la
Teoría General de Sistemas (Rodríguez-Jiménez y Pérez-Jacinto, 2017) que recomienda el método documental sistémico-estructural-funcional tratándose de organizaciones, el cual se ejemplifica en la siguiente figura 2:

\section{Figura 2}

Fases del método de investigación sistémico-estructural-funcional

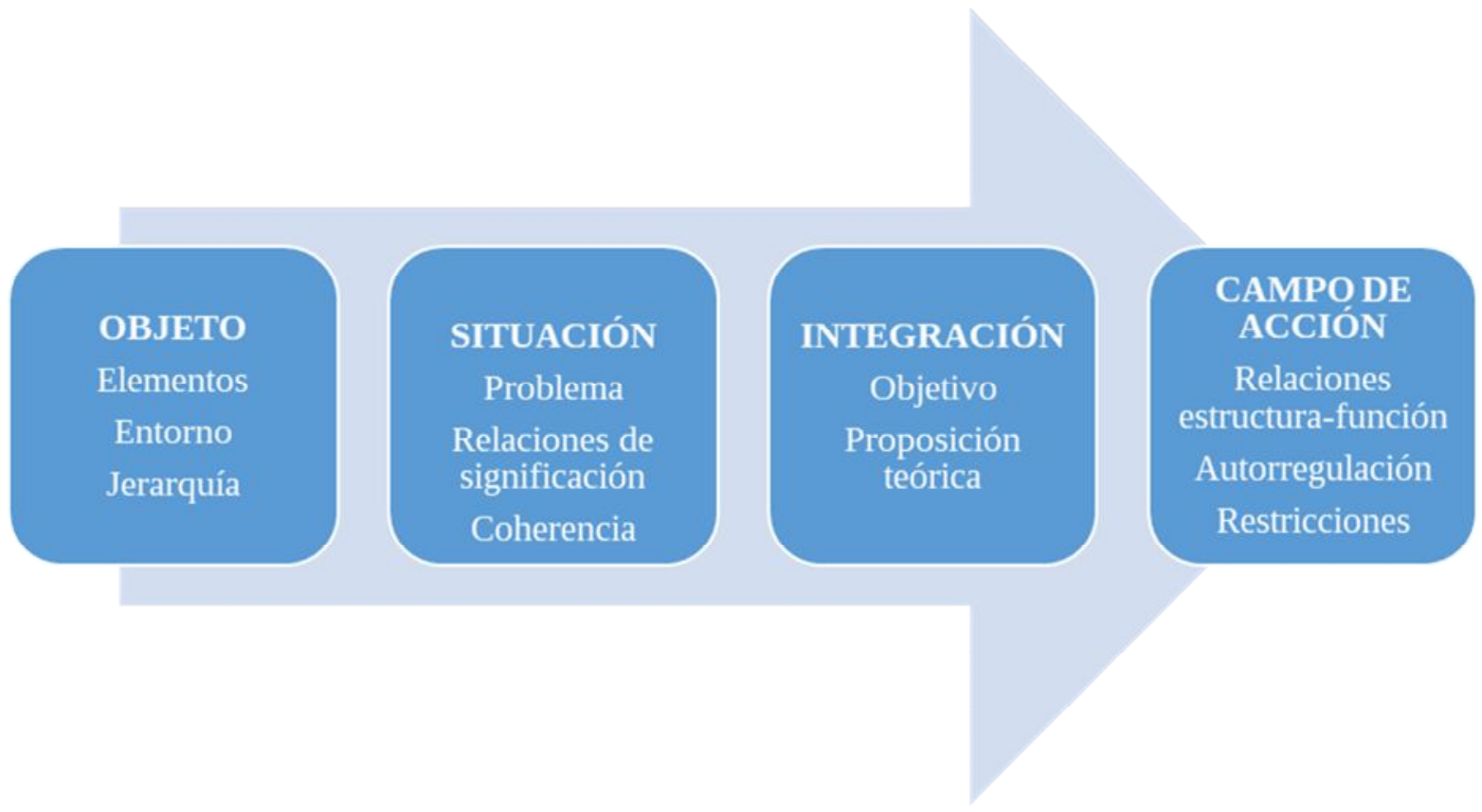

Fuente: Elaboración propia.

Como primer paso se construyó un marco teórico de referencia, caracterizando la figura del emprendedor y las actividades educativas relacionadas con su fomento, habiéndose identificado sus elementos componentes y el contexto en el que se dan (ver figura 3); enseguida se analizó la situación identificando el problema mediante el uso de relaciones de significación y coherencia, hasta concluir con las preguntas que detonan la investigación: ¿cuáles actividades se promueven en las universidades para desarrollar el talento emprendedor? y ¿qué actividades se consideran más efectivas para lograrlo? Posteriormente se integró toda la información del hecho perceptible en análisis, construyendo el siguiente objetivo de investigación: identificar las actividades que promueven el emprendimiento en los estudiantes de Ingeniería en Gestión Empresarial del ITSMante; el último paso es la delimitación del campo de estudio, en el que se identifican las relaciones del perfil de IGE con las actividades educativas que promueven el emprendimiento, con la limitante de estar restringidas 
solamente a esta carrera. Una vez realizado lo anterior, se propone: "Las actividades que promueven el emprendimiento en los estudiantes de IGE del ITSMante no son suficientes para desarrollar las competencias emprendedoras".

\section{Figura 3}

Análisis de las actividades de fomento al emprendimiento en IGE

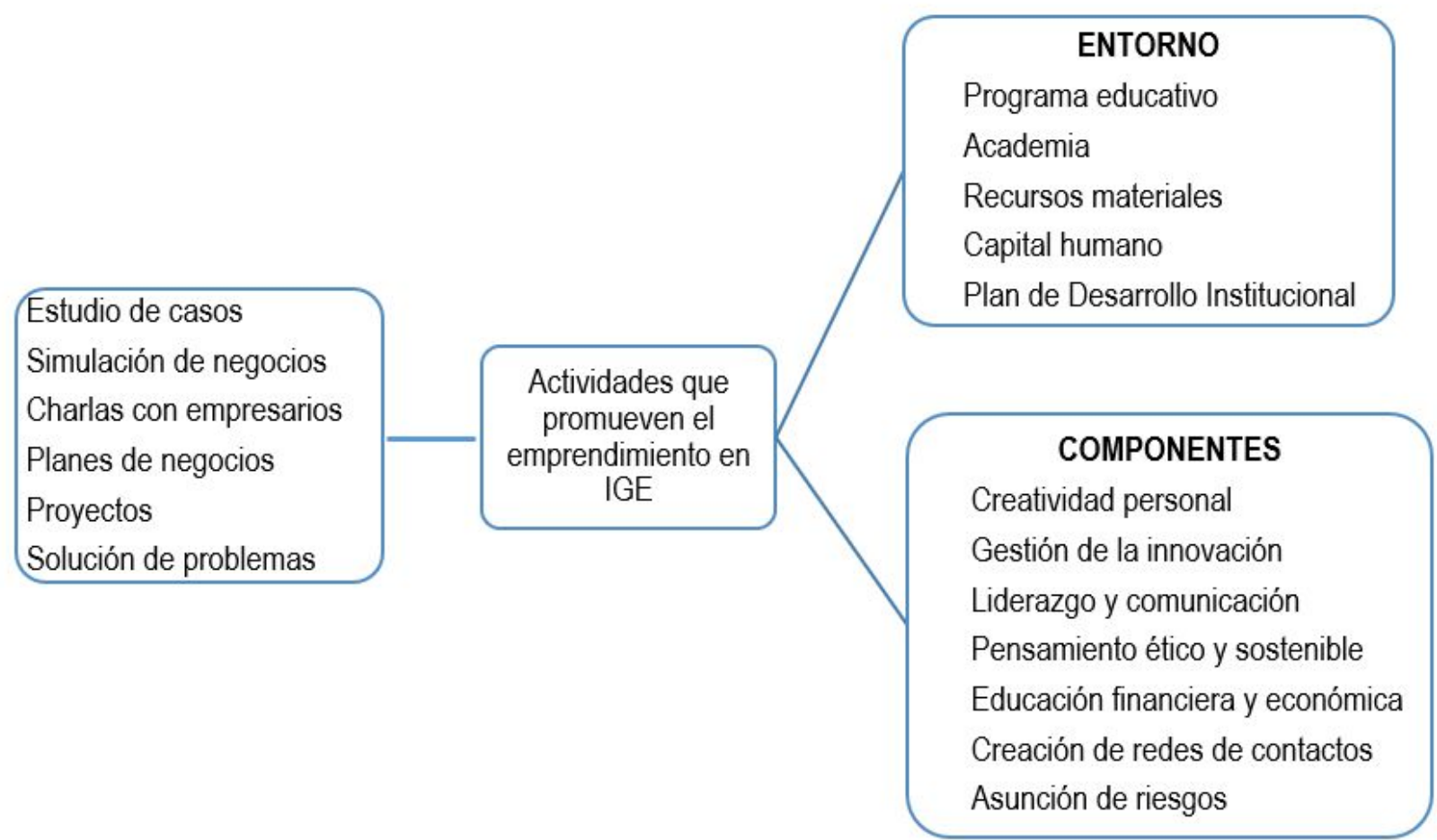

\section{Resultados}

\section{EI perfil de egreso de la carrera de Ingeniería en Gestión Empresarial}

El plan de estudios de una carrera contiene el perfil de egreso profesional, las competencias, los conocimientos y habilidades, así como las actitudes y valores que se pretende formar en el educando; las competencias representan "la integración y aplicación estratégica de conocimientos, procedimientos y actitudes necesarios para la solución de problemas, con una actuación profesional ética, eficiente y pertinente en escenarios laborales heterogéneos y cambiantes” (Tecnológico Nacional de México, 2015). Las competencias son las 
herramientas fundamentales que le permitirán al egresado su fácil inserción en el mundo laboral, demandante de profesionistas con una identidad profesional cimentada y competencias preferentes en investigación, dominio de un segundo idioma y capacidad de comunicación oral y escrita, esto a partir de investigaciones en el contexto empresarial mexicano (Fernández-Macas e Iglesias-León, 2016; Páez, 2013).

\section{Tabla 1}

Competencias del perfil de egreso de la carrera de Ingeniería en Gestión Empresarial.

1. Desarrollar y aplicar habilidades directivas y la ingeniería en el diseño, creación, gestión, desarrollo, fortalecimiento e innovación de las organizaciones, con una orientación sistémica y sustentable para la toma de decisiones en forma efectiva.

2. Diseñar e innovar estructuras administrativas y procesos, con base en las necesidades de las organizaciones para competir eficientemente en mercados globales.

3. Gestionar eficientemente los recursos de la organización con visión compartida, con el fin de suministrar bienes y servicios de calidad

4. Aplicar métodos cuantitativos y cualitativos para el análisis e interpretación de datos y modelado de sistemas, en los procesos organizacionales para la mejora continua, atendiendo estándares de calidad mundial.

5. Diseñar, evaluar y emprender nuevos negocios y proyectos empresariales, que promuevan el desarrollo sustentable y la responsabilidad social, en un mercado competitivo.
6. Diseñar e implementar estrategias de mercadotecnia basadas en información recopilada de fuentes primarias y secundarias del consumidor o usuario de algún producto, de acuerdo a oportunidades y amenazas del mercado.

7. Establecer programas para el fortalecimiento de la seguridad e higiene en las organizaciones.

8. Gestionar sistemas integrales de calidad, ejerciendo un liderazgo efectivo y un compromiso ético, aplicando las herramientas básicas de la ingeniería.

9. Interpretar y aplicar normas legales que incidan en la creación y desarrollo de las organizaciones.

10. Integrar, dirigir y desarrollar equipos de trabajo para la mejora continua y el crecimiento integral de las organizaciones.

11. Analizar e interpretar la información financiera para detectar oportunidades de mejora e inversión en un mundo global, que incidan en la rentabilidad del negocio. 


\section{Tabla 1}

\section{Continuación...}

12. Utilizar las nuevas tecnologías de información en la organización, para optimizar los procesos de comunicación y eficientar la toma de decisiones.

13. Propiciar el desarrollo del capital humano, para la realización de los objetivos organizacionales, dentro de un marco ético y un contexto multicultural.
14. Aplicar métodos de investigación para desarrollar e innovar sistemas, procesos y productos en las diferentes dimensiones de la organización.

15. Gestionar la cadena de suministros de las organizaciones con un enfoque orientado a procesos.

16. Analizar e interpretar la economía global para facilitar la toma de decisiones en la organización.

Fuente: TecNM (2015).

Primeramente se hizo necesario analizar detenidamente los enunciados del Perfil de Egreso de la carrera de Ingeniería en Gestión Empresarial que se imparte en el Instituto Tecnológico Superior de El Mante (ver tabla I), con el propósito de identificar las competencias relacionadas con el emprendimiento; a partir de un trabajo teórico-conceptual colegiado de la academia basado en el análisis grupo nominal se lograron reconocer aquellas competencias que se relacionan con las habilidades para el diseño y la creación de nuevas empresas; de las 16 competencias que busca formar la institución en los estudiantes de esta carrera, al menos dos están relacionadas con el emprendimiento: la primera, que está enfocada en el desarrollo de habilidades directivas para diseñar y crear una organización, y la quinta, enfocada en darle el carácter sustentable y de responsabilidad social a nuevos negocios y proyectos empresariales; estas dos características son deseables en un profesionista que será gestor de empresas, para lo cual la participación del docente es esencial, siendo que el problema focal en la educación empresarial es la formación de estas competencias de emprendimiento, y es aquí cuando el profesor se convierte en agente transformador de la realidad del educando (De la Torre Cruz et al., 2016).

Como siguiente paso se construyó un listado de las competencias que inciden en el emprendimiento, de entre las propuestas por Estrada de la Cruz, Mira Solves y Gómez Gras (2016) y por Paños Castro (2017), seleccionadas a conveniencia del autor aunque sin valorar aún la efectividad de las mismas; en la literatura revisada se estudian con detenimiento cada una de ellas, por lo que no se consideró necesario agregar sus descripciones en este trabajo y solamente se utilizaron para construir la tabla II siguiente; de igual forma, entre las metodologías o actividades más utilizadas para la promoción del emprendimiento se documentan en las fuentes consultadas en las bases de datos científicas las presentaciones, workshops, juegos y competiciones, la creación de empresas reales, los proyectos, la creación 
de planes de negocios, invitados emprendedores, proyección de videos y películas, simulaciones de empresas, debates y trabajo en grupo, estudio de casos, conferencias, la teoría y algunas otras similares.

A partir de las competencias seleccionadas anteriormente y de las actividades que fomentan el emprendimiento se ha realizado un trabajo para indagar al interior de la organización cuáles son las actividades dentro del programa institucional de IGE que inciden en la adquisición de las competencias mencionadas, así como la frecuencia de tales actividades, los participantes y la opinión de la Academia sobre la efectividad o bondad de su implementación, quedando plasmados los resultados en la Tabla II siguiente:

\section{Tabla 2}

Relación de actividades emprendedoras en el programa de IGE

\begin{tabular}{|c|c|c|c|c|}
\hline Competencias & $\begin{array}{c}\text { Actividades en el programa } \\
\text { IGE }\end{array}$ & Frecuencia & Participantes & Evaluación \\
\hline \multirow{3}{*}{$\begin{array}{l}1 \text { Creatividad } \\
\text { personal }\end{array}$} & $\begin{array}{l}\text { Feria de emprendedores } \\
\text { Se integran equipos que } \\
\text { desarrollan un proyecto } \\
\text { empresarial. }\end{array}$ & $\begin{array}{l}\text { Un evento al } \\
\text { año }\end{array}$ & Toda la carrera & $\begin{array}{l}\text { Reconocido por todos } \\
\text { como el que más } \\
\text { aporta a desarrollar la } \\
\text { creatividad personal. }\end{array}$ \\
\hline & $\begin{array}{l}\text { Plan de negocios } \\
\text { Se elabora un plan en la } \\
\text { materia del mismo nombre } \\
\text { pero no se lleva a la } \\
\text { práctica el negocio. }\end{array}$ & $\begin{array}{l}\text { Una vez al } \\
\text { año }\end{array}$ & $\begin{array}{l}\text { Grupos de } \\
\text { semestre V }\end{array}$ & $\begin{array}{l}\text { Se exige como } \\
\text { requisito en la Feria } \\
\text { de emprendedores. }\end{array}$ \\
\hline & $\begin{array}{l}\text { Taller de Investigación I } \\
\text { y II } \\
\text { Donde elaboran proyectos } \\
\text { y prototipos creativos. }\end{array}$ & $\begin{array}{l}\text { Dos veces al } \\
\text { año }\end{array}$ & $\begin{array}{c}\text { Grupos de } \\
\text { semestres V y VI }\end{array}$ & $\begin{array}{l}\text { Origina participación } \\
\text { en concursos de } \\
\text { creatividad e } \\
\text { innovación } \\
\text { tecnológica. }\end{array}$ \\
\hline
\end{tabular}




\section{Tabla 2}

Continuación...

\begin{tabular}{|c|c|c|c|c|}
\hline Competencias & $\begin{array}{c}\text { Actividades en el programa } \\
\text { IGE }\end{array}$ & Frecuencia & Participantes & Evaluación \\
\hline \multirow{2}{*}{$\begin{array}{l}2 \text { Gestión de la } \\
\text { innovación }\end{array}$} & $\begin{array}{l}\text { Clínicas empresariales } \\
\text { Se realiza vinculación con } \\
\text { empresas para dar solución } \\
\text { a problemas de diverso } \\
\text { ámbito. }\end{array}$ & $\begin{array}{l}\text { Un evento al } \\
\text { año }\end{array}$ & $\begin{array}{c}\text { Grupos de } \\
\text { semestre VI }\end{array}$ & $\begin{array}{l}\text { Reconocido porque se } \\
\text { invita al empresario a } \\
\text { la evaluación final. }\end{array}$ \\
\hline & $\begin{array}{l}\text { Metodología para la } \\
\text { solución de problemas } \\
\text { Materia nueva. }\end{array}$ & $\begin{array}{l}\text { Una vez al } \\
\text { año }\end{array}$ & $\begin{array}{c}\text { Grupos de } \\
\text { semestre VIII }\end{array}$ & $\begin{array}{l}\text { Aun no evaluado en } \\
\text { otro contexto. }\end{array}$ \\
\hline \multirow{2}{*}{$\begin{array}{l}3 \text { Liderazgo y } \\
\text { comunicación }\end{array}$} & $\begin{array}{l}\text { Concurso de oratoria } \\
\text { La temática suele ser } \\
\text { elegida por autoridades } \\
\text { locales }\end{array}$ & $\begin{array}{l}\text { Un evento al } \\
\text { año }\end{array}$ & $\begin{array}{l}\text { Participación } \\
\text { voluntaria }\end{array}$ & $\begin{array}{l}\text { Escasa participación, } \\
\text { se elige un } \\
\text { representante de la } \\
\text { institución }\end{array}$ \\
\hline & $\begin{array}{l}\text { Jóvenes emprendedores } \\
\text { Es un curso ofrecido por un } \\
\text { organismo municipal }\end{array}$ & $\begin{array}{l}\text { Una vez al } \\
\text { año }\end{array}$ & $\begin{array}{l}\text { Participación } \\
\text { voluntaria }\end{array}$ & $\begin{array}{l}\text { Escasa participación } \\
\text { por el costo para el } \\
\text { alumno }\end{array}$ \\
\hline $\begin{array}{l}4 \text { Creación de } \\
\text { redes de contactos }\end{array}$ & $\begin{array}{l}\text { No se tiene ninguna } \\
\text { actividad en esta categoría }\end{array}$ & & & \\
\hline $\begin{array}{l}5 \text { Pensamiento } \\
\text { ético y sostenible }\end{array}$ & $\begin{array}{l}\text { Actividades curriculares } \\
\text { Comprendidas en varias } \\
\text { materias }\end{array}$ & Todo el año & Toda la carrera & $\begin{array}{l}\text { Está incluido en el } \\
\text { programa de IGE }\end{array}$ \\
\hline
\end{tabular}




\section{Tabla 2}

\section{Continuación...}

\begin{tabular}{|c|c|c|c|c|}
\hline Competencias & $\begin{array}{c}\text { Actividades en el programa } \\
\text { IGE }\end{array}$ & Frecuencia & Participantes & Evaluación \\
\hline \multirow{3}{*}{$\begin{array}{l}6 \text { Educación } \\
\text { financiera y } \\
\text { económica }\end{array}$} & $\begin{array}{l}\text { Actividades curriculares } \\
\text { Comprendidas en varias } \\
\text { materias }\end{array}$ & Todo el año & Toda la carrera & $\begin{array}{l}\text { Está incluido en el } \\
\text { programa de IGE }\end{array}$ \\
\hline & $\begin{array}{l}\text { Jóvenes emprendedores } \\
\text { Descrita en la competencia } \\
3\end{array}$ & & & \\
\hline & $\begin{array}{l}\text { Taller de finanzas } \\
\text { Se ofrece en los congresos } \\
\text { locales }\end{array}$ & $\begin{array}{l}\text { Una vez al } \\
\text { año }\end{array}$ & $\begin{array}{c}\text { Participación por } \\
\text { niveles }\end{array}$ & $\begin{array}{l}\text { Con enfoque en las } \\
\text { finanzas y la } \\
\text { economía }\end{array}$ \\
\hline \multirow{3}{*}{$\begin{array}{l}7 \text { Asunción de } \\
\text { riesgos }\end{array}$} & $\begin{array}{l}\text { Feria de emprendedores } \\
\text { Descrita en la competencia } \\
1\end{array}$ & & & \\
\hline & $\begin{array}{l}\text { Incubadora de negocios } \\
\text { ITSM } \\
\text { Herramienta para quienes } \\
\text { desean emprender y } \\
\text { necesitan apoyo }\end{array}$ & $\begin{array}{l}\text { Estará abierta } \\
\text { todo el año }\end{array}$ & $\begin{array}{l}\text { Alumnos, } \\
\text { residentes y } \\
\text { egresados }\end{array}$ & $\begin{array}{l}\text { Sin experiencia por } \\
\text { ser la primera vez que } \\
\text { se ofrece }\end{array}$ \\
\hline & $\begin{array}{l}\text { Dreams Factory } \\
\text { Se promueve la fabricación } \\
\text { de productos para ofrecerse } \\
\text { en un evento al fin del } \\
\text { ciclo, arriesgando el capital } \\
\text { invertido. }\end{array}$ & $\begin{array}{l}\text { Un evento al } \\
\text { año }\end{array}$ & $\begin{array}{l}\text { Grupos de } \\
\text { semestre VI }\end{array}$ & $\begin{array}{l}\text { Sin experiencia por } \\
\text { ser la primera vez que } \\
\text { se ofrece }\end{array}$ \\
\hline
\end{tabular}

Fuente: Elaboración propia. 
El trabajo de análisis permitió identificar al menos 7 competencias directamente relacionadas con el espíritu emprendedor que se busca despertar en los estudiantes de Gestión Empresarial, basadas en las características que demuestran los jóvenes emprendedores y ello a partir del trabajo bibliométrico realizado, encontrándose los siguientes resultados:

La primera de ellas, la creatividad personal, es la que más distingue a los estudiantes de esta carrera y se promueve en al menos 3 actividades pertinentes que se realizan durante el año escolar: la feria de emprendedores es el evento principal de la agenda y está presente en el programa de la academia desde el año 2011, el aprendizaje del plan de negocios se perfecciona en una materia del mismo nombre y el taller de investigación se ha convertido en semillero para trabajos y proyectos con destino a los concursos de ciencias, creatividad e innovación.

En el caso de la gestión de la innovación, una actividad ad-hoc del programa son las clínicas empresariales, donde los alumnos resuelven problemáticas reales de diversa índole y presentan sus resultados frente a un panel que incluye al empresario; en otra asignatura de reciente creación, que ocurre después de esta actividad, los alumnos son enseñados a enfrentar la solución de problemas con diferentes metodologías científicas.

Respecto al liderazgo y comunicación la academia percibe que dichas competencias son genéricas y están contempladas en las actividades curriculares, particularmente en los primeros semestres, además de concursos de oratoria en la fase local; otras actividades que apuntalan el emprendimiento en este tema son los talleres de emprendedores que imparte una entidad municipal, con la anotación de que, al no ser un evento gratuito para el alumno, se desincentiva su participación, motivo por el que hay una escasa participación.

La creación de una red de contactos que permita o incentive el emprendimiento es una tarea olvidada por la academia y la misma requiere de un trabajo colaborativo, apoyándose en las áreas de vinculación de la universidad con las empresas y con centros de promoción empresarial del sector gobierno, incluso del tercer sector; como respuesta de los docentes a esta omisión, se ha tomado nota para buscar la inclusión de alguna actividad en el programa anual de trabajo.

La quinta competencia que apuntala el espíritu emprendedor es el pensamiento ético y sostenible, dado que impacta directamente con las competencias emprendedoras que se han identificado en la primera parte de esta sección: coincidentemente es la quinta competencia del perfil de IGE, que busca darle el carácter sustentable y de responsabilidad social a nuevos negocios y proyectos empresariales; esta cualidad del egresado está contemplada en el programa curricular de estudios, donde las actividades de enseñanzaaprendizaje son diseñadas para resaltar la importancia de la conducta ética en el ejercicio de la profesión, así como el quehacer sostenible en todos los ámbitos de su desempeño.

La siguiente competencia resalta la importancia de desarrollar las habilidades directivas del educando: el conocimiento en el área financiera y económica de las empresas se promueve desde las asignaturas del plan de estudios, pero también se hace con los talleres que organiza la academia en forma extracurricular, como serían los que se imparten durante los congresos multidisciplinarios o durante la semana de la ingeniería, 
en el caso de esta institución tecnológica.

La última y no menos importante competencia seleccionada destaca la trascendencia de la asunción de riesgos por el emprendedor: se promueve en las ferias, en concursos, pero también se buscará estimularla con dos nuevas actividades: se ha iniciado una incubadora de negocios con el modelo del Tecnológico Nacional de México para cobijar a 9 proyectos en forma inicial en agosto 2020, cuyos resultados aún no están a la vista; además, se promueve un concurso adaptado de las cooperativas escolares del Programa EME del Principado de Asturias (Sobrado Fernández y Fernández Rey, 2010), pero adaptado al nivel superior, donde el capital invertido será puesto en juego en una sola oportunidad de comercialización.

\section{Conclusiones}

El trabajo de análisis permitió la identificación de algunas de las competencias del programa emprendedor de IGE con los pilares del ecosistema emprendedor señalado anteriormente; se encuentran los pilares habilidades (2) y soporte cultural (5), los cuales deben ser reforzados como tarea del sistema educativo; también el pilar capital humano (8) es impulsado en este programa en estudio, el cual está enfocado en el desarrollo de las habilidades directivas y para ello se integran actividades que aumentan la experiencia del alumnado y su capacidad de innovar; además, se relacionan con los pilares innovación del producto (10) e innovación del proceso (11) que se establecen como resultado de la capacidad creativa e innovadora del estudiante.

Por otro lado, las competencias nivel de tecnología (7), competencia (9) e internacionalización (13) han sido calificadas como valores exógenos al programa ya que no dependen del estudiante sino de otras estructuras del ecosistema emprendedor; de igual forma se identificó que la competencia networking (4) no ha sido promovida aún en ninguna actividad, por lo que es necesario desarrollar esta capacidad del estudiante para que pueda relacionarse con otros y comparta o amplíe su conocimiento empresarial.

También se analizaron las actividades que recién se integran en el programa emprendedor y cuya efectividad no ha sido evaluada aún; éstas se relacionan con las competencias oportunidad empresarial (1) y aceptación o aversión al riesgo (3), junto con la competencia emprendimiento oportuno (6), y las competencias alto crecimiento (12) y capital de riesgo (14), ya que se buscará que el alumno pueda encontrar el momento propicio para realizar sus proyectos sin temor y con el menor riesgo con las dinámicas propuestas, tanto la incubadora de negocios como el Dream Factory, para lo cual es pertinente la búsqueda por parte de la Academia de un fondo de capital semilla para que los estudiantes puedan acceder al financiamiento de sus proyectos.

En un ejercicio de visualización radial a partir de los resultados anteriores se utilizó una escala ideal de 4 puntos sobre las actividades y los contenidos del programa curricular que detonan el emprendimiento, y se observa que en el programa emprendedor del ITSMante sólo se potencian la creatividad personal y la educación financiera y económica del estudiante, dejando de lado las demás competencias que se han identificado como necesarias para lograr el perfil emprendedor que se busca en el estudiante de la carrera de Ingeniería en Gestión Empresarial; lo anterior confirma la proposición teórica ofrecida inicialmente, ya que las actividades actuales implementadas por la Academia son insuficientes para promover el perfil emprendedor, al no ser cubiertas todas las competencias específicas que integran dicho perfil, tal como se observa en la figura 4 siguiente; particularmente es nula la inclusión de actividades que promuevan la creación 
de una red de contactos o empresas y tampoco hay un programa de liderazgo y comunicación o de asunción de riesgos, por lo que se reconoce un resultado general de 2 sobre el ideal de 4 .

\section{Figura 4}

Actividades y competencias emprendedoras de IGE en el ITSMante

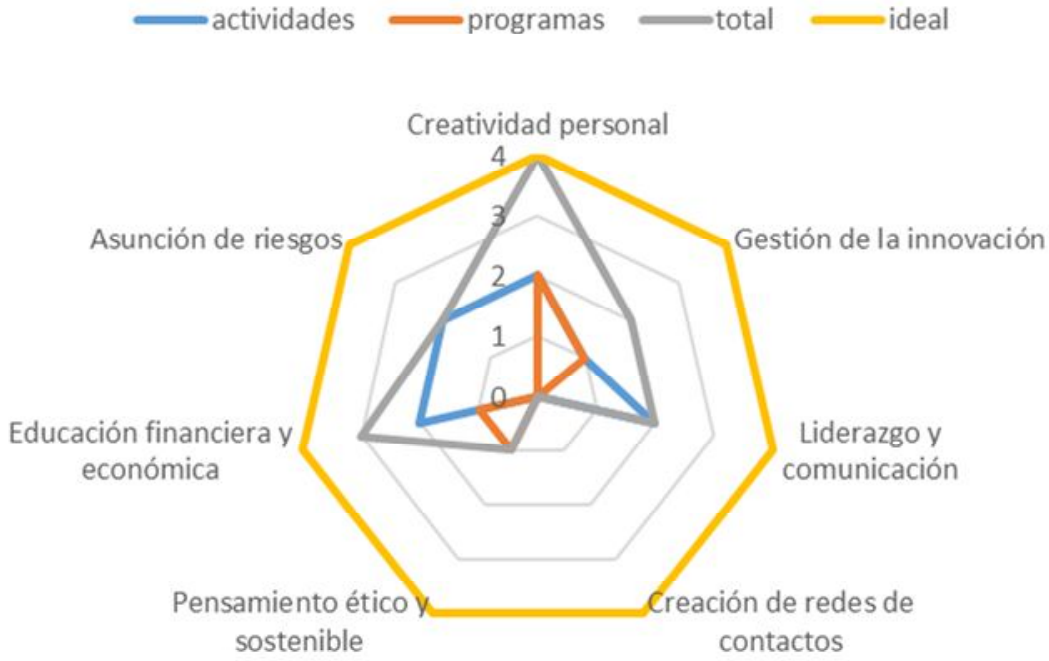

Fuente: Elaboración propia.

Aunque las actividades del programa emprendedor de IGE sí permiten desarrollar algunas habilidades que se buscan en las dos competencias estudiadas del perfil de egreso de la carrera, se reconoce la limitación de su efectividad ya que con ellas no se logra una formación integral en emprendimiento, tal como sucede con los programas de otras universidades.

\section{Discusión}

En el análisis de la literatura sobre el emprendimiento se ha reconocido que numerosos estudios señalan la falta de consenso sobre la génesis del emprendedor, desde sus características hasta los factores que le motivan a emprender; al respecto, Herrera Guerra y Montoya Restrepo (2013) nos señalan que el fenómeno debe analizarse en su contexto original y debe observarse como parte de un ecosistema mediante el pensamiento sistémico, esto concuerda con lo señalado por Ács et al. (2020) que fundamentan su trabajo en el ecosistema emprendedor mostrado antes en la figura 1; un elemento de dicho ecosistema con particular importancia es el sistema educativo, que como hemos señalado es el 
encargado de promover el emprendimiento mediante 3 formas habituales: educando sobre el emprendimiento, para el emprendimiento y a través del emprendimiento (Sánchez García Ward, Hernández y Florez, 2017); las universidades suelen realizar esta actividad utilizando el enfoque de crear actividades independientes de los programas académicos en la forma de actividades extracurriculares, aunque Marulanda Valencia, Montoya Restrepo y Vélez Restrepo (2014) abogan por lo opuesto: sugieren que se incluyan como asignaturas para enseñar la gestión, supervivencia y desarrollo de pequeñas y grandes industrias por igual.

Los programas formales de emprendedores son un desafío y a la vez una tarea indispensable para las universidades, y su desarrollo e implementación son tan importantes como su mantenimiento (Sánchez García et al., 2017); el diseño de cada actividad requiere analizar el impacto que tendrá en las competencias específicas que se quieren alcanzar en los estudiantes y que tienen relación con el emprendimiento; aunque hay diversidad de opiniones en la literatura del tema, las competencias básicas de creatividad, innovación, responsabilidad ética e iniciativa emprendedora se encuentran entre las más mencionadas en el tema del emprendimiento, y son las que se han podido identificar en el programa emprendedor del ITSMante analizado en este trabajo (Peña Calvo, Cárdenas Gutiérrez, Rodríguez Martín y Sánchez Lissen, 2015).

Para lograr en los estudiantes las competencias emprendedoras se requieren métodos activos de enseñanza diferentes a los establecidos en los programas formativos, como sugieren Sánchez García et al. (2017), quienes también afirman que se requiere formar a los docentes en tales métodos de enseñanza, de ahí la importancia de este trabajo documental; son deseables aquellos centrados en solución de problemas, desarrollo de proyectos y trabajo colaborativo; una actividad muy interesante que vale la pena incorporar en los programas emprendedores es la que comparten Orozco Triana, Parra Bernal, Ruíz Arias y Matíz Bulla (2016): fortalecer las habilidades de comunicación de los jóvenes mediante talleres grupales (Elevator Pitch). Los métodos de enseñanza deben enfocarse en desarrollar los rasgos asociados con el éxito empresarial, siendo de gran influencia para despertar el espíritu emprendedor de los jóvenes la instrucción de las ventajas del emprendimiento, como son las recompensas económicas y personales, la independencia o autonomía, la seguridad familiar y personal o conseguir un patrimonio personal (Rueda Sampedro, Fernández-Laviada y Herrero Crespo, 2013); si los estudiantes no cuentan con un soporte cultural en su ecosistema que aprecie el emprendimiento individual, serán impulsados a permanecer en el modelo tradicional de educación formal y búsqueda de empleo (Valencia Arias et al., 2015).

A pesar de la aparente efectividad de los programas emprendedores universitarios, es preciso medir su impacto en las actitudes y competencias emprendedora de los estudiantes, ya que se ha demostrado que éstas mejoran con métodos de enseñanza que se apegan al modelo emprendedor (Sánchez-García y HernándezSánchez, 2016), por lo que se sugiere continuar este estudio hasta identificar los beneficios que aporta al perfil de egreso del Ingeniero en Gestión Empresarial.

\section{Referencias}

Ács, Z. J., Szerb, L., Lafuente, E., y Márkus, G. (2020). The Global Entrepreneurship Index 2019. The Global Entrepreneurship and Development Institute. 
Almanza Jiménez, R., y Vargas-Hernández, J.G. (2015). Las Competencias Profesionales y su relación con la empleabilidad de los Ingenieros en Gestión Empresarial egresados del ITLAC. Revista Electrónica Gestión de las Personas y Tecnología, 8(22), 17-28.

Bedoya Villa, M. A., y Arango Alzate, B. (2017). Orientación Emprendedora, Recursos y Capacidades: Una Revisión de su Marco Conceptual para la Innovación. Espacios, 38, 11-29.

Carlos Ornelas, C. E., Contreras González, L. E., Silva Olvera, M. A., y Liquidano Rodríguez, M. C. (2015). El Espíritu Emprendedor y un Factor que Influencia su Desarrollo Temprano. Conciencia Tecnológica, 49, 46-51.

De la Torre Cruz, T., Luis Rico, I., Escolar Llamazares, C., Palmero Cámara, C., y Jiménez Eguizábal, A. (2016). La figura del profesor como agente de cambio en la configuración de la competencia emprendedora. Revista Interuniversitaria de Formación del Profesorado, 86, 131-144.

Estrada de la Cruz, M., Mira Solves, I., y Gómez Gras, J. M. (2016). El entorno universitario como generador de capacidades emprendedoras: un análisis de percepciones. En M. T. Tortosa Ybáñez, S. Grau Company y J. D. Álvarez Teruel (Eds.), Investigación, innovación y enseñanza universitaria, enfoques pluridisciplinares (pp. 2926-2946). Instituto de Ciencias de la Educación (ICE) Universidad de Alicante.
Fernández Macas, H., e Iglesias León, M. (2016). Análisis de la integración de teoría y la práctica de la disciplina de administración de operaciones en la formación de administradores de empresas, reflexión desde lo académico y laboral. Revista Universidad y Sociedad, 8(1), 50-58.

Fuentes García, F. J., y Sánchez Cañizares, S. M. (2010). Análisis del perfil emprendedor: una perspectiva de género. Estudios de Economía Aplicada, 28(3), 1-27.

Garzozi, R., Messina, M., Moncada, C., Ochoa, J., Ilabel, G., y Zambrano, R. (2014). Planes de Negocios para Emprendedores (1a ed.). Iniciativa Latinoamericana de Libros de Texto Abiertos (LATIn).

Guerrero, M., y Urbano, D. (2012). Transferencia de conocimiento y tecnología. Mejores prácticas en las universidades emprendedoras españolas. Gestión y Política Pública XXI, 107-139.

Herrera Guerra, C. E., y Montoya Restrepo, L. A. (2013). El emprendedor: una aproximación a su definición y caracterización. Revista Punto de Vista, IV(7), 7-30.

Il Sung Park, S., y Duarte Masi, S. (2015). El perfil del emprendedor y los estudios relacionados a los emprendedores Iberoamericanos. Revista Internacional de Investigación en Ciencias Sociales, 11(2), 298-314.
Vol. 6, núm. 17 / mayo - agosto del 2021 DOI: https://doi.org/10.36791/tcg.v0i17.100 
Leite, E., Correia, E., Sánchez-Fernández, M., y Leite, E. (2015). El espíritu emprendedor: condicionantes para la innovación. HOLOS, Año 31, 5, 278-291.

Marulanda Valencia, F. Á., Montoya Restrepo, I. A., y Vélez Restrepo, J. M. (2014). Aportes teóricos y empíricos al estudio del emprendedor. Cuadernos de Administración, 30(51), 89-99.

Moreno Zacarías, H. M., Espíritu Olmos, R., y Priego Huertas, H. (2013). La presencia de las aptitudes emprendedoras en las Universidades públicas de México. En J. Sánchez Gutiérrez (Ed.), La arquitectura financiera en las organizaciones para la competitividad (pp. 1711-1729). Red Internacional de Investigadores en Competitividad.

Moriano León, J. A., Palací Descals, F. J., y Morales Domínguez, J. F. (2006). El perfil psicosocial del emprendedor universitario. Revista de Psicología del Trabajo y de las Organizaciones, 75-99.

Orozco Triana, J. A., Parra Bernal, L. D., Ruíz Arias, C. L., y Matíz Bulla, F. J. (2016). Acompañamiento a emprendedores en la Universidad EAN: Del modelo IN3 a EAN Impacta -2011-2016-. Revista Escuela de Administración y Negocios, 81, 45-74.

Páez, M. (2013). Estudio de Vinculación Empresas-IES. Síntesis Ejecutiva. Alianza FIIDEM AC.

Paños Castro, J. (2017). Educación emprendedora y metodologías activas para su fomento. Revista electrónica interuniversitaria para la formación del profesorado, 33-48.
Peña Calvo, J., Cárdenas Gutiérrez, A., Rodríguez Martín, A., y Sánchez Lissen, E. (2015). La cultura emprendedora como objetivo educativo: marco general y estado de la cuestión. En L. Núñez Cubero (Ed.), Cultura emprendedora y educación (pp. 19-59). Editorial Universidad de Sevilla.

Rodríguez Jiménez, A., y Pérez Jacinto, A. O. (2017). Métodos científicos de indagación y construcción del conocimiento. Revista Escuela de Administración y Negocios, 82, 179-200.

Rueda Sampedro, I., Fernández-Laviada, A., y Herrero Crespo, Á. (2013). Aplicación de la teoría de la acción razonada al ámbito emprendedor en un contexto universitario. Investigaciones Regionales, 26, 141-158.

Sánchez García, J. C., Ward, A., Hernández, B., y Florez, J. L. (2017). Educación emprendedora: Estado del arte. Propósitos y Representaciones, 5(2), 401-437.

Sánchez-García, J. C., y Hernández-Sánchez, B. R. (2016). Influencia del Programa Emprendedor Universitario (PREU) para la mejora de la actitud emprendedora. Pampa, 13, 55-75.

Sánchez Rodríguez, S., Hernández Herrera, C. A., y Jiménez García, M. (2016). Análisis de la percepción sobre iniciativa empresarial y el espíritu emprendedor en estudiantes de un tecnológico federal. Acta Universitaria, 26(6), 70-82.

Sobrado Fernández, L., y Fernández Rey, E. (2010). Competencias emprendedoras y desarrollo del espíritu empresarial en los centros educativos. Educación XX1, 13(1), 15-38. 
Tecnológico Nacional de México. (2015). Manual de Lineamientos Académico-Administrativos del TECNM. Ciudad de México: Tecnológico Nacional de México.

Valencia Arias, J. A., Montoya Restrepo, I., y Montoya Restrepo, A. (2015). Factores explicativos de las intenciones emprendedoras en estudiantes universitarios. Espacios, 36, 7-16.
Vargas Hernández, J. G., y Campos Álvarez, R. E. (2011). Habilidades emprendedoras en los jóvenes universitarios zacatecanos. Revista ECORFAN, 2(5), $70-90$

Zachman, P., López, W., y Redchuk, A. (2015). Aproximación y relevancia de la creación de empresas de Base Tecnológica Universitarias en Argentina. Revista Iberoamericana de Educación en Tecnología y Tecnología en Educación, 15, 63-72. 